\title{
Cost-effectiveness of strategies to control the spread of carbapenemase- producing Enterobacterales in hospitals: A modelling study
}

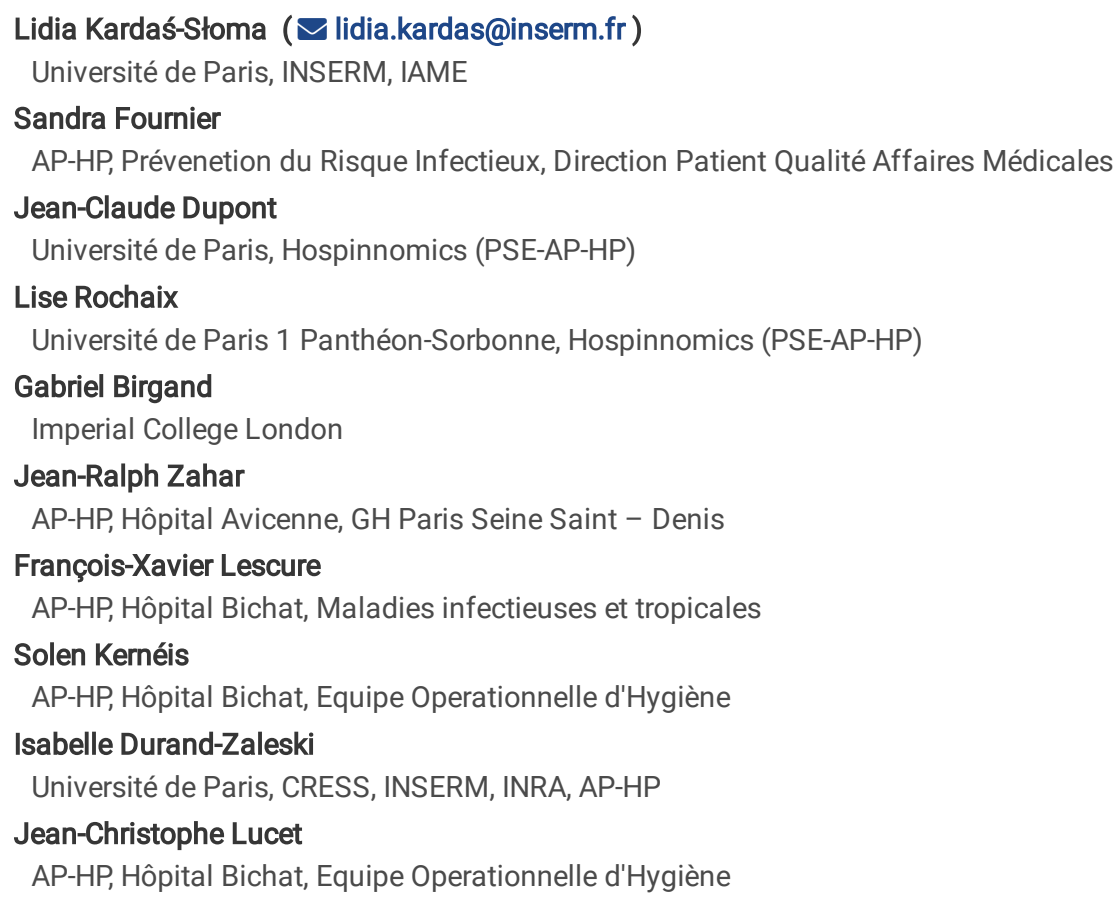

\section{Research Article}

Keywords: Cross-transmission, Carbapenemase-producing Enterobacterales, Hand disinfection, Mathematical model, Cost-effectiveness, Control strategies, France

Posted Date: January 24th, 2022

DOI: https://doi.org/10.21203/rs.3.rs-1276558/v1

License: (c) (i) This work is licensed under a Creative Commons Attribution 4.0 International License. Read Full License 


\section{Abstract}

\section{Background}

Spread of resistant bacteria causes severe morbidity and mortality. Stringent control measures can be expensive and disrupt hospital organization. In the present study, we assessed the effectiveness and cost-effectiveness of control strategies to prevent the spread of Carbapenemase-producing Enterobacterales (CPE) in a general hospital ward (GW).

\section{Methods}

A dynamic, stochastic model simulated the transmission of CPE by the hands of healthcare workers (HCWs) and the environment in a hypothetical 25bed GW. Input parameters were based on published data; we assumed the prevalence at admission of $0.1 \%$.

12 strategies were compared to the baseline (no control) and combined different prevention and control interventions: targeted or universal screening at admission (TS or US), contact precautions (CP), isolation in a single room, dedicated nursing staff (DNS) for carriers and weekly screening of contact patients (WSC). Time horizon was one year. Outcomes were the number of CPE acquisitions, costs, and incremental cost-effectiveness ratios (ICER).

A hospital perspective was adopted to estimate costs, which included laboratory costs, single room, contact precautions, staff time, i.e. infection control nurse and/or dedicated nursing staff, and lost bed-days due to prolonged hospital stay of identified carriers. The model was calibrated on actual datasets. Sensitivity analyses were performed.

\section{Results}

The baseline scenario resulted in $0.93 \mathrm{CPE}$ acquisitions/1000 admissions and costs $32,050 € / 1000$ admissions. All control strategies increased costs and improved the outcome.

The efficiency frontier was represented by: 1) TS with DNS at a 17,310 €/avoided CPE case, 2) TS + DNS + WSC at a $26,322 € /$ avoided CPE case and 3) US + DNS + WSC at 179,839 €/avoided CPE case. Other strategies were dominated.

Sensitivity analyses showed that TS + CP might be cost-effective if CPE carriers are identified upon admission or if the cases have a short hospital stay. However, CP were effective only when high level of compliance with hand hygiene was obtained.

\section{Conclusions}

Targeted screening at admission combined with DNS for identified CPE carriers with or without weekly screening were the most cost-effective options to limit the spread of CPE. These results support current recommendations from several high-income countries.

\section{Introduction}

Carbapenemase-producing Enterobacterales (CPE) are increasingly common in hospitals and represent a serious health problem. These multidrugresistant organisms colonise the gastrointestinal tract after direct (person-to-person) or indirect (via contaminated surfaces) transmission. Klebsiella Pneumoniae and Escherichia coli are the common causes of urinary tract infections, ventilator-associated pneumonia and bloodstream infections in healthcare settings [1]. In 2015, in the EU/ EEA, the annual number of CPE infections was estimated as 15,947 for K. Pneumoniae and 2,619 for E. coli [2]. Treatment options for patients infected with CPE are limited, leading to high mortality, and increased length of stay and hospital costs. The successful implementation of cost-effective infection control measures to prevent CPE spread and infections is key for hospital managers.

Recommendations to limit the transmission of CPE in healthcare facilities are based on the early detection of asymptomatic carriers, implementation of contact precautions and isolation in a single room [3-6]. In practice, strategies combining various interventions are employed according to the risk assessment and available resources: 1) universal or targeted rectal screening on admission, 2) standard precautions (SP), applied to all patients regardless of their infectious status, 3) contact precautions (CP) for identified carriers or infected patients, 4) isolation in a single room, 5) environmental cleaning, 6) rectal screening of contact patients, i.e. those whose care was provided by the same team as the CPE patient, and/or 7 ) isolation of carriers in a dedicated area with dedicated nursing staff (DNS), hereafter designated as cohorting.

However, these control measures pose challenges such as the high cost of patient screening and cohorting, requirement for single room isolation or staff shortage for implementing precautions. Moreover, the effectiveness and cost-effectiveness of various CPE control strategies is underdocumented [4].

Mathematical models can be used to study the effectiveness, cost and cost-effectiveness of control strategies and to help decision-makers in the identification of the best combination of interventions to control the spread of antimicrobial-resistant organisms. These models make it possible to test the potential impact of different interventions before real-world implementation, which can save time and resources. Nevertheless, stringent strategies for controlling CPE have rarely been evaluated through mathematical modelling [7-10]. 
Our objective was to compare the impact, cost and cost-effectiveness of different strategies combining screening and contact precaution measures to control the spread of CPE in a general medicine ward (GW) using a mathematical model.

\section{Methods}

\section{Model}

We used a compartmental, stochastic model [11,12] to describe the dynamics of CPE transmission in a general medicine ward (GW). The model simulates hospital patient admission and discharge, the spread of a CPE between patients via contact with healthcare workers (HCWs) and the hospital environment. In this model, patients are classified as either uncolonised, colonised- unidentified, colonised-identified or infected. HCWs can be uncontaminated or transiently contaminated (hands) (Figure 1). Appendix A1 provides details of the model.

\section{Assumptions and parameters of model}

We modelled a 25-bed GW, with the continuous presence of $10 \mathrm{HCWs}$ (5 nurses and 5 nursing assistants)[13-15]. At t0, we assumed a CPE-free ward and simulated the admission and discharge of patients. Bed occupancy was assumed to be $100 \%$. The prevalence of CPE carriage at the time of hospital admission varies widely between countries but remains low in high-income Western-European countries [16,17]. We assumed the baseline prevalence of CPE carriage on admission to be $0,1 \%$ [17-20]. Other scenarios for CPE carriage on admission were considered in a sensitivity analysis.

The model parameters are presented in Table 1. We used data from the literature and from university hospital trusts operating in Paris and its surroundings (AP-HP). Each day, each patient received an average of $28 \mathrm{HCW}$ visits (or 2.8 visits per HCW per day) [13,14]. During a contact with a colonised patient, an HCW could contaminate hands with CPE with a probability of $b_{h}=0.21$ [7] and transmit bacteria to other patients. Uncolonised patients could become colonised (intestinal colonisation) after contact with a contaminated HCW. The unknown probability of intestinal colonisation $\left(b_{p}\right)$ was calibrated in order to reproduce an average number of secondary cases of 0.77 per patient (range between 0 and 3 ), observed in a multicentre study from three hospitals [21]. Our model was also calibrated on ESBL-PE from a large interventional European multicentre study, considering that CPE spread might match ESBL spread [22]. The parameter $b_{p}$ obtained from this calibration was used in a sensitivity analysis.

We assumed that uncolonised patients could also become colonised with CPE through the hospital environment at rate $a=0.00001$ per day [23-25]. As CPE colonisation can persist for months [26], we assumed that patients who acquired CPE remained colonised during their hospital stay. The average length of stay (LOS) of uncolonised patients was set at 6 days. Colonisation and infection with CPE have been reportedly associated with increased hospital stay [27-29]. We assumed that LOS of infected or colonised-identified patients was 25 days, giving an excess LOS of 3 weeks in comparison with uncolonised patients as reported in other studies [27,29]. The average LOS of colonised patients non-identified during hospital stay was estimated at 12 days [21]. In the model, the probability that a CPE-colonised person would develop symptomatic infection during hospital stay was 0.076 [30]. The probability of death during hospital stay for uncolonised, colonised and infected patients was $0.020,0.036$ and 0.3 respectively [31-33].

Table 1 Input parameters and plausible ranges for sensitivity analysis. 


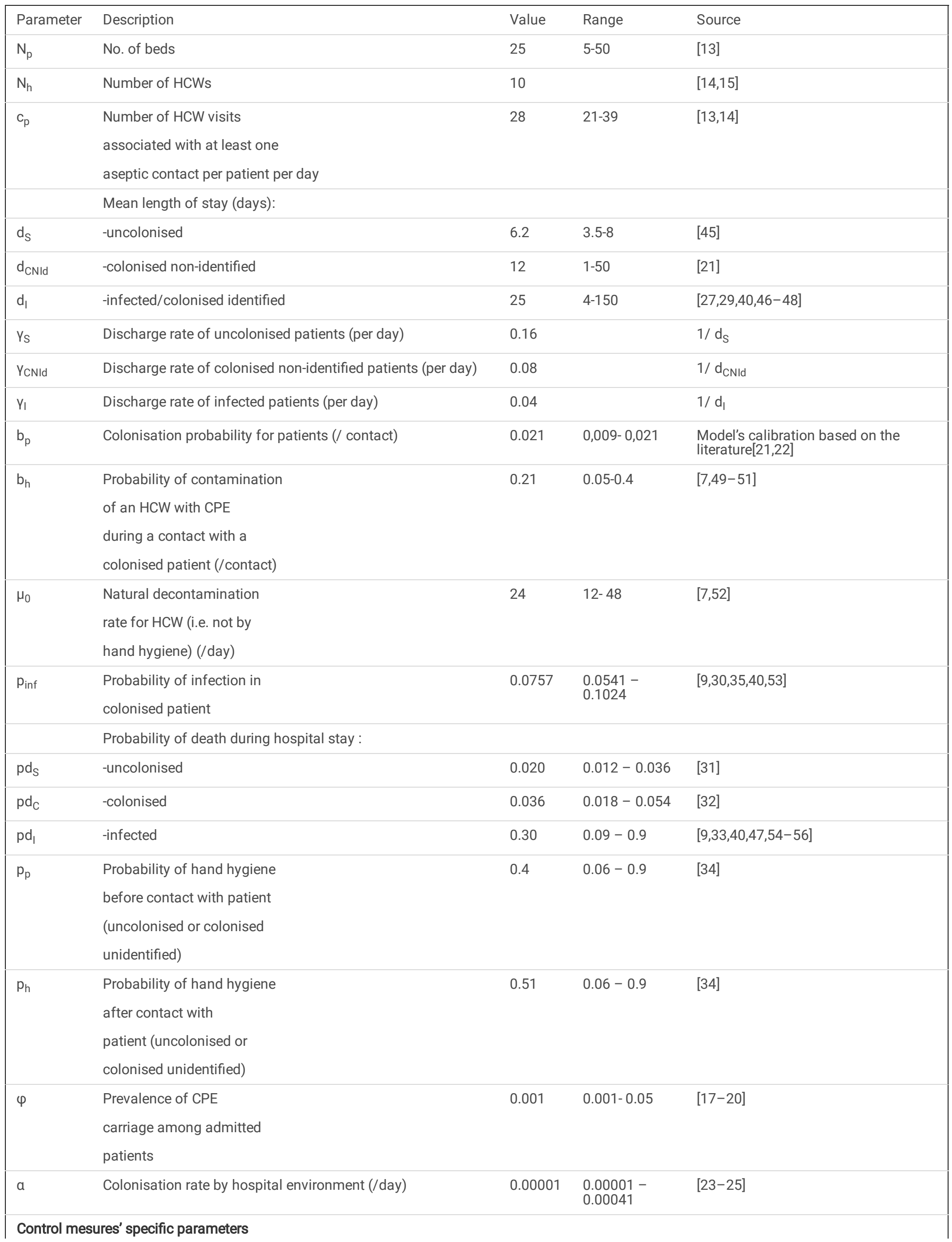




\begin{tabular}{|lllll|}
\hline & $\begin{array}{l}\text { Part of colonised patients, identified at admission in a risk- } \\
\text { based screening }\end{array}$ & 0.5 & $0.2-0.5$ & Local data and [17,35] \\
\hline$r_{\mathrm{a}}$ & Sensitivity of the screening method (\%) & 0.96 & $0.9-1$ & [57-59] \\
\hline $\mathrm{s}_{\mathrm{p}}$ & Specificity of the screening method (\%) & 1 & 0.8 & assumed \\
\hline $\mathrm{p}_{\mathrm{id}}$ & Probability of hand hygiene & & \\
\hline $\mathrm{r}_{\mathrm{s}}$ & before/after contact with identified CPE patient & 10 & assumed \\
\hline
\end{tabular}

\section{Baseline scenario}

In the baseline scenario, we assumed the following : standard contact precautions with $40 \%$ hand hygiene (HH) compliance before contact with a patient and $50 \%$ after patient contact [34], no rectal screening on admission and no additional contact measures.

\section{Infection control strategies}

Twelve strategies were gradually implemented and compared to the baseline scenario.

The first six strategies combined targeted or universal screening at admission and control measures applied to identified CPE carriers:

1) Targeted screening (TS) + contact precautions (CP) without isolation of carriers in a single room,

2) $\mathrm{TS}+\mathrm{CP}+$ isolation in a single room,

3) TS + dedicated nursing staff (DNS) + isolation of carriers in a single room,

4) Universal screening (US) + CP without isolation in a single room,

5) US + CP + isolation in a single room,

6) US + DNS + isolation in a single room.

Further strategies (7-12) consisted in the enforcement of previous control measures through the weekly screening of contact patients (WSC), that is, patients cared for by the same HCWs as the CPE-positive patient.

Targeted screening was defined as the screening of patients with a history of CPE infection or colonisation, patients with a history of foreign hospital stay within the last year and patients repatriated from a hospital abroad [4-6]. We estimated from the literature and from personal experience that current risk-based screening at hospital admission would identify $50 \%$ of CPE-positive patients [17, 35].

In the universal screening strategy, all admitted patients were screened with a rapid screening test (e.g. PCR) and a result obtained within $24 \mathrm{~h}$. The positive PCR test had to be confirmed by culture, which was included in costs. Weekly screening was based on culture only. For simplicity, we assumed $100 \%$ specificity. The compliance with $\mathrm{HH}$ before/after contact with a patient was $80 \% / 80 \%$ in the strategy with contact precautions. This value corresponds to the upper bounds of the interval of $\mathrm{HH}$ compliance reported in several studies [34,36].

\section{Costs}

The analysis was performed from a public hospital perspective. The cost of hospital day was estimated using the French severity-adjusted, diagnosisrelated group. Other costs were drawn from the literature and from previous studies of our team [11,18,37]. Cost were expressed in 2021 Euro $(1 €=1.19 \$)$.

The cost of the baseline scenario (reference strategy) was considered to be the cost of $\mathrm{HH}$ at baseline level (cost of the alcohol-based hand rub and staffing time) and cost of extended stay for CPE infected patients.

The costs of control measures were split into: 1) cost of rectal screening (testing materials and laboratory costs) and culture for CPE confirmation, 2) cost of contact precautions (gown, gloves, improved HH, infection team staff time), 3) cost of single room isolation (if necessary, transformation of a double-room into a single-arranged room, with the resulting loss in revenue for the hospital due to "blocked beds" and reduced admissions), 4) cost of dedicated nursing staff, and 5) cost of extended stay of identified CPE carriers. See Table 2 in the Appendix A1 for more details and for cost parameters (Table 2).

\section{Model simulations and outcomes}

We ran the model over a 1-year period to capture all costs and health effects relevant to control strategies implemented. 
Simulations of the model were performed using Gillespie's method and programmed in C++ language. The outcomes (number of CPE acquisitions, cost of intervention and incremental cost-effectiveness ratio (ICER; the ratio of the difference in costs to difference in health benefits)) were calculated after a period of 1 year and as an average of 5000 Monte Carlo simulations.

\section{Cost-effectiveness evaluation}

In the cost-effectiveness analysis, we estimated the costs associated with each strategy and the health benefits expressed as the number of CPE cases (acquisitions) avoided when compared with the baseline scenario. We eliminated all dominated strategies, i.e. strategies that had higher cost and provided lower health benefit than one other strategy or a combination of two other strategies. For the non-dominated strategies, we calculated the incremental cost-effectiveness ratios (ICER) calculated as the extra annual cost of one strategy relative to another divided by CPE cases avoided by that strategy relative to the other strategy. Finally, we constructed an efficiency frontier comparing more costly, but more effective strategies.

\section{Sensitivity analysis}

We performed several additional analyses to assess the impact of our assumptions and parameter uncertainty on the model's outcomes.

\section{Deterministic sensitivity analysis}

We first ran a univariate sensitivity analysis to consider the impact of a lower compliance with $\mathrm{HH}(60 \% / 60 \%)$ in strategies with $\mathrm{CP}$. Then we investigated the model with reduced LOS of identified CPE cases (reduction by 50\%) and also a reduced LOS of one day for all categories of patients (that could be considered as home hospitalisation). We also considered 1) a better identification of colonised patients in a risk-based screening at admission ( $90 \%$ vs $50 \%$ in central analysis), 2) higher prevalence of CPE carriage at admission (from $0.1 \%$, to $1 \%$ or $5 \%$ ) with less patients presenting with risk factors for CPE colonisation (20\%). We then ran the model with a lower probability of colonisation, based on the another calibration[22].

We also performed a cost-effectiveness analysis with the cost of a hospital bed day higher than our baseline value (900 $€$ vs $500 €$ ). We ran the model with a modified initial condition and assumed that a CPE identified carrier was present in a ward at t0 (in the central analysis we had a CPE-free ward at $\mathrm{t0}$ ). Finally, we compared the effectiveness of standard precautions with the targeted screening + contact precautions for a different the level of $\mathrm{HH}$ compliance.

\section{Probabilistic sensitivity analysis}

We performed a probabilistic sensitivity analysis to explore the effect of joint uncertainty across parameters (except strategy-specific parameters that were fixed) on the cost-effectiveness of strategies. In this analysis, we used triangular distributions for epidemiological or healthcare organisation parameters and gamma distributions for healthcare costs.

Then, we represented the cost-effectiveness acceptability curves graphically, showing the probability of each strategy having the highest net monetary benefit at different values of willingness to pay for a CPE case avoided.

\section{Results}

For the strategy with standard contact precautions (baseline), over one year, $0.93 \mathrm{CPE}$ acquisitions per 1,000 admissions occurred. Among the cases, $92 \%$ were colonised from HCWs contaminated hands and $8 \%$ from the environment.

Compared to the baseline, all strategies were effective in limiting the spread of CPE (Figure 2).

Strategies combining universal screening (US) with contact precautions (CP) or dedicated staff (DNS) reduced the number of cases by $22-98 \%$ and were more effective than those with targeted screening (TS) (reduction of 16-64\%). Weekly screening (WSC) helped identify the additional number of CPE carriers but had little impact on nosocomial spread.

The most effective strategies combined screening on admission with dedicated staff for identified carriers. In strategy 3 (TS + DNS), we observed 0.34 CPE acquisitions / 1000 admissions (reduction of $64 \%$ compared to the baseline). In strategy 6 (US + DNS), there were 0.02 cases / 1000 admissions (reduction of $98 \%$ ). Strategies with weekly screening + DNS reduced CPE acquisition by $66 \%$ and $98 \%$ respectively.

Isolation of carriers in a single room + CP (strategies $2,5,8,11$ ) reduced the number of CPE acquisitions from $27 \%$ to $35 \%$ depending on the screening scenario chosen.

The least effective strategy combined screening with CP without single room isolation of identified carriers (strategies $1,4,7,10)$. The reduction in cases ranged from $16 \%$ to $22 \%$ depending on the screening scenario.

\section{Cost-effectiveness of control strategies}

The mean total cost of the baseline scenario was the lowest and estimated at $€ 32,050 / 1000$ admissions (Table 2). 
Table 2 Results of cost-effectiveness analysis. TS- targeted screening; US- universal screening; CP- contact precautions; DNS- dedicated staff; WSweekly screening.

\begin{tabular}{|c|c|c|c|c|c|c|}
\hline Strategy & $\begin{array}{l}\text { Total cost } / 1000 \\
\text { admissions } \\
\text { (€) }\end{array}$ & $\begin{array}{l}S D \\
(€)\end{array}$ & $\begin{array}{l}\text { Nb of CPE } \\
\text { acquisitions } / 1000 \\
\text { admissions }\end{array}$ & $\begin{array}{l}\Delta \text { Cost } / 1000 \\
\text { admissions }(€)\end{array}$ & $\begin{array}{l}\triangle \mathrm{Nb} \text { of CPE } \\
\text { acquisitions } / 1000 \\
\text { admissions }\end{array}$ & $\begin{array}{l}\text { ICER } \\
\text { (€/avoided } \\
\text { case) }\end{array}$ \\
\hline Baseline & 32050 & 2443 & 0.93 & & & \\
\hline 1. $\mathrm{TS}+\mathrm{CP}$ & 37304 & 5567 & 0.78 & & & Dominated* \\
\hline $\begin{array}{l}\text { 2. } \mathrm{TS}+\mathrm{CP}+\text { single } \\
\text { room }\end{array}$ & 37509 & 5636 & 0.68 & & & Dominated* \\
\hline $\begin{array}{l}\text { 8. TS+ CP + single } \\
\text { room + WSC }\end{array}$ & 38455 & 6866 & 0.66 & & & Dominated* \\
\hline 7. $T S+C P+W S C$ & 38560 & 7285 & 0.78 & & & Dominated* \\
\hline 3. TS + DNS & 42320 & 10916 & 0.33 & 10270 & 0.59 & 17310 \\
\hline 9. TS + DNS + WSC & 42934 & 11641 & 0.31 & 614 & 0.02 & 26322 \\
\hline 4. US + CP & 86165 & 6716 & 0.72 & & & Dominated* \\
\hline $\begin{array}{l}\text { 11.US + CP + single } \\
\text { room + WSC }\end{array}$ & 87151 & 7931 & 0.62 & & & Dominated* \\
\hline 10.US + CP+ WSC & 87231 & 8245 & 0.72 & & & Dominated* \\
\hline $\begin{array}{l}\text { 5. US + CP + single } \\
\text { room }\end{array}$ & 87345 & 7204 & 0.60 & & & Dominated* \\
\hline 6. US + DNS & 95427 & 13446 & 0.02 & & & Dominated* \\
\hline $\begin{array}{l}\text { 12.US + DNS + } \\
\text { WSC }\end{array}$ & 95561 & 13553 & 0.02 & 52627 & 0.29 & 179839 \\
\hline
\end{tabular}

*Dominated: a strategy is dominated when it has higher cost and provides lower health benefit than one other strategy or a combination of two other strategies.

Each control strategy led to health gains compared to the baseline but required higher resource utilization. The cost of strategies ranged from $€ 37,304 / 1000$ admissions to $€ 95,561 / 1000$ admissions with the most expensive strategies including US (strategies 4-6 and 10-12) (Table 2). In general, $84 \%$ to $90 \%$ of the cost was due to the implementation of control measures (additional personnel, screening, etc.) and $10 \%$ to $16 \%$ to the loss of hospital revenue due to the extended stay of identified carriers.

In the cost-effectiveness analysis, the efficiency frontier of prevention of CPE transmission was represented by strategies: 3 ) TS + dedicated nursing staff (DNS) + single room, 9) TS + DNS + single room + WSC, and 12) US+ DNS + single room + WSC (Figure 3). Other strategies were dominated.

The cost of moving from the base case to the strategy 3) TS + dedicated nursing staff (DNS) + single room was €17,310/ avoided CPE case. Moving from the strategy 3 to 9) TS + DNS + single room + WSC costs an additional €26,322/ avoided case. Finally, moving from the strategy 9 to 12) US + DNS + single room + WSC costs €179,839/avoided case.

\section{Deterministic sensitivity analysis}

Findings from the sensitivity analysis confirmed the cost-effectiveness of strategies with dedicated staff for scenarios with: a reduced LOS of hospitalised patients, a lower probability of colonisation, a higher prevalence of CPE carriage at admission, a higher prevalence at admission combined with the lower identification of carriers by a risk-based screening, a CPE case identified at admission, and a higher cost of a hospital bedday.

We have also found, that targeted screening combined with single room isolation of carriers and implementation of contact precautions were costeffective if CPE carriers were identified upon admission or if the cases had a short stay in the ward. However, contact precautions were effective only when high level of compliance with $\mathrm{HH}$ was obtained. The summary, and details of results from these analyses, are presented in the Appendix A2.

\section{Probabilistic sensitivity analysis}

Results are presented as cost-effectiveness acceptability curves (Figure 4), showing the probability of each strategy having the highest net monetary benefit at different values of willingness-to-pay threshold. 
For example, at willingness-to-pay values lower than $€ 675$ per case avoided, strategy 1 . TS + CP had the highest probability of being cost-effective (50$75 \%)$, followed by strategy 2 . TS + CP + single room. At willingness-to-pay $€ 17,000-25,000$, strategy 3 . TS + DNS is an optimal option, in balance with strategy 9. TS + DNS + WSC and strategy 2. Finally, above $€ 25,000$ per case averted, strategy 9 became optimum.

\section{Discussion}

The implementation of strategies to prevent the spread of CPE must take into consideration both costs and health benefits. In order to assist decision makers, we used a mathematical model of CPE transmission in a general medicine ward and evaluated the cost-effectiveness of twelve different strategies. We found that the targeted screening of at-risk patients at admission combined with dedicated staff for identified CPE carriers with or without weekly screening was the most cost-effective option.

Our findings show that in the baseline scenario, over one year, $0.93 \mathrm{CPE}$ acquisitions per 1,000 admissions occurred. From an individual perspective, the likelihood of acquiring CPE (and infection) in a ward seems low. Nevertheless, from a national hospital perspective or from a societal perspective, the emergence of CPE remains worrying and can lead to outbreaks that are more difficult to manage, threaten patient's safety, impact clinical services and are more costly to control than invested costs at the initial step of the epidemics $[18,27,38]$.

We have shown that all strategies modelled were effective in reducing the number of CPE acquisitions. This reduction ranged from $16 \%$ to $98 \%$ depending on the intervention and was the greatest for strategies with dedicated staff. Our results are consistent with other studies evaluating the effectiveness of control measures to limit the spread of highly resistant bacteria [39-41]. For example, Fournier et al. [40] have shown that the introduction of a control program in a large hospital network was very effective in reducing the number of secondary transmissions, despite the increasing number of CPE index cases. This study also underlined the importance of the early implementation of interventions after the identification of cases and the importance of dedicated staff.

Our results show that adding weekly screening had little impact on the nosocomial spread of CPE. This could be explained by the low prevalence of carriage at admission in our model. However, in real-life situations, where the cases are often discovered accidently during the hospital stay, weekly screening may help to identify additional CPEs and is important in the management of outbreaks.

In our study, the introduction of control strategies increased the cost by $16 \%$ to $34 \%$ (for strategies with targeted screening) and $169 \%$ to $198 \%$ (for strategies with universal screening) compared to baseline. In terms of cost-effectiveness, the efficiency frontier of CPE prevention was represented by strategies combining targeted or universal screening at admission with dedicated staff. We also showed that the ICER increased steeply with universal screening. With a current prevalence of CPE carriage in Western-European countries (less than 1\%), the willingness to pay for strategy 12 is rather hypothetical. The deterministic sensitivity analysis showed that if the prevalence is higher (e.g. 1-5\%) or if the identification of carriers by a risk-based screening is weak, strategy 12 becomes more cost-effective (Appendix A2, Table A6 and A7). These results are in accordance with another modelling study which found that universal screening could be cost-effective, depending on the prevalence of CPE colonization in admitted patients [9].

The implementation of a control strategy with dedicated staff (in a context of constrained budget and human resources) is a challenge for the hospital. According to data from Public Health France in 2019, dedicated staff were employed one out of one hundred times for the management of a CPE case discovered at admission and one out of ten times when a case was identified during the hospital stay [42]. Given the major impact of dedicated staff for controlling CPE spread, instituting cohorting of CPE carriers from different units within a single area appears desirable, at least in large hospitals affected by CPE. This measure was also included in the CPE management recommendations [4-6].

We investigated other hypotheses to validate the robustness of our predictions and to find conditions under which CP might be a cost-effective option. We showed that TS+ CP+ single room could be cost-effective when a CPE case was identified upon admission or when a case had a shorter stay in the ward ( 12 days instead of 25 days in the central analysis), but only if a high level of compliance with $\mathrm{HH}$ ( $80 \%-80 \%)$ was obtained. Accelerating the transfer of CPE patients from acute care stay to dedicated rehabilitation units could be cost-effective.

Despite the recommendations [4-6] and the confirmed effectiveness of $\mathrm{HH}$ in prevention of nosocomial infections, compliance with $\mathrm{HH}$ remains low and often lower than the values used in our baseline scenario (40/50\% before/after contact). In another analysis, we varied the level of HH and compared SP with the effectiveness of TS + CP. We found that a high HH compliance in SP might be even more effective than TS + CP (Appendix A2, Figure 1). This high level of compliance can be registered during audits of compliance with $\mathrm{HH}$, but is actually rarely achieved when the compliance measurement is unobtrusive, with 2- to 2.5-fold lower compliance than through direct observation[43, 44]. However, improving compliance with HH must remain a central objective in controlling CPE spread, especially in hospitals where dedicated staff or cohorting is not an available option.

We also tested the realistic strategy of a higher prevalence of CPE carriage on admission (1\%), with carriers presenting risk factors in only $20 \%$ of cases. Here again, the strategies with dedicated staff remained optimal, suggesting that these strategies should be used, even with an evolution towards an endemic CPE situation.

Our study has several strengths. First, we used a dynamic model to consider that the risk of colonisation depends on the number of carriers and can change over time. It also allows testing the effectiveness of interventions under different hypotheses (e.g. prevalence of CPE at admission). Second, our model was calibrated on various datasets, on CPEs [21] but also on ESBL-PE from a large European multicentre study [22], considering that CPE spread may not be different from ESBL spread. In addition, our study examined a range of control strategies combining various scenarios for both

Page 8/15 
screening at admission as well as management of cases in the ward. Such an assessment using traditional epidemiological tools based on large, cluster-randomised studies is difficult, can be impacted by bias and does not make it possible to distinguish the individual impact of interventions.

In addition to the above points, our model has also taken into consideration the possibility that uncolonised patients could become colonised with CPE through the hospital environment. This way of transmission is rarely included in modelling studies. Finally, we tested the effectiveness and costeffectiveness of strategies under different hypotheses and studied the impact of uncertainty in the estimation of the model's parameters on our predictions. We also chose a hospital perspective which, while not recommended by international guidelines, is the most likely to convince hospital managers. While the healthcare system perspective considers all production costs, the hospital perspective looked at both cost and revenues, which is what hospital managers do in systems driven by a DRG-like prospective payment. This analysis also allowed us to find the conditions under which the strategy based on CP was cost-effective, or to show that strategies with dedicated staff were the best options even if the prevalence of carriers at admission was higher.

Our study also has several limitations. First, we did not model the interruption of new admissions and transfers in an outbreak situation, as recommended by several guidelines. In our simulations, the situation of an outbreak was rare (occurring in about $4 \%$ of simulations), but we acknowledge that bed closures could represent the highest costs to contain an outbreak [18, 27].

Another potential limitation relates to a paucity of evidence about the length of stay for each patient category: either colonised-unidentified, identified or infected. In the literature, the additional length of stay of identified CPE cases (colonised identified/infected) compared to non-carrier patients was approximately 3 weeks $[27,29]$ and could be explained by the difficulties in transferring cases to downstream units. It is not clear whether the LOS of colonised-unidentified patients is longer than non-carriers. A multicentre study reported a LOS of unidentified colonised patients, incidentally discovered post-hospitalization, twice as long as the average LOS in the medical ward [21]. This extended LOS may be linked to other factors that promote CPE acquisition, e.g. intensity of care or exposure to antibiotics. However, patient LOS is an important parameter for the model, with a strong impact on transmission and costs.

Finally, costs were estimated based on local and national data and may not be generalizable to other countries.

\section{Conclusions}

In conclusion, our findings suggest that the targeted screening of at-risk patients at admission, combined with dedicated staff for identified CPE carriers with or without weekly screening, was the most cost-effective strategy to control the spread of CPE in a ward. This result holds true even though the prevalence of CPE carriage at admission is high and in variable sensitivity analyses. Targeted screening combined with isolation of carriers in a single room and implementation of contact precautions may merit consideration if CPE carriers are identified upon admission or if the cases have a short stay in the ward. However, contact precautions are effective only when a high level of compliance with $\mathrm{HH}$ is obtained.

\section{Abbreviations}

AP-HP- university hospital trusts operating in Paris and its surroundings

CP- contact precautions

CPE- Carbapenemase-producing Enterobacterales

DNS- dedicated nursing staff

GW- in a general hospital ward

HCWs- healthcare workers

$\mathrm{HH}$ - hand hygiene

ICER- incremental cost-effectiveness ratio

LOS- length of stay

TS- targeted screening

US- universal screening

WSC- weekly screening of contact patients

\section{Declarations}

Ethics approval and consent to participate 
Not applicable.

\section{Consent for publication}

Not applicable.

\section{Availability of data and materials}

All data generated or analysed during this study are included in this article and its supplementary information files.

\section{Competing interests}

All authors declare no competing interests.

\section{Funding}

This work was supported by the ARS Ile de France and by the French government 's PREPS program (grant no. 19-0109).

\section{Authors' contributions}

JCL, SF, IDZ, and LKS designed the study. LK, SF, IDZ, and JCL conducted the data acquisition. LKS, SF, JCD, LR, GB, JRZ, FXL, SK, IDZ, and JCL analysed data needed for parameterisation. LKS did the modelling and computer simulations. LKS, SF, JCD, LR, GB, JRZ, FXL, SK, IDZ, and JCL contributed to interpretation, drafting and critical revision of the work, and final approval of the manuscript.

\section{Acknowledgements}

We thank Lulla Opatowski and Laura Temime for useful discussion on our model's design and calibration.

\section{References}

1. Patients: Information about CRE [Internet]. 2021 [cited 2021 Dec 7]. Available from: https://www.cdc.gov/hai/organisms/cre/cre-patients.html

2. Cassini A, Högberg LD, Plachouras D, Quattrocchi A, Hoxha A, Simonsen GS, et al. Attributable deaths and disability-adjusted life-years caused by infections with antibiotic-resistant bacteria in the EU and the European Economic Area in 2015: a population-level modelling analysis. Lancet Infect Dis. 2019;19:56-66.

3. Magiorakos AP, Burns K, Rodríguez Baño J, Borg M, Daikos G, Dumpis U, et al. Infection prevention and control measures and tools for the prevention of entry of carbapenem-resistant Enterobacteriaceae into healthcare settings: guidance from the European Centre for Disease Prevention and Control. Antimicrobial Resistance \& Infection Control. 2017;6:113.

4. World Health Organization. Guidelines for the prevention and control of carbapenem-resistant Enterobacteriaceae, Acinetobacter baumannii, and Pseudomonas aeruginosa in health care facilities. [Internet]. 2017 [cited 2021 Oct 1]. Available from: http://www.ncbi.nlm.nih.gov/books/NBK493061/ 5. Haut Conseil de la Santé Publique. Actualisation des recommandations relatives aux BHRe [Internet]. 2019. Available from: https://www.hcsp.fr/Explore.cgi/avisrapportsdomaine?clefr=758

6. Public Health England. Framework of actions to contain carbapenemase-producing Enterobacterales [Internet]. 2020 [cited 2021 Nov 15]. Available from: https://assets.publishing.service.gov.uk/government/uploads/system/uploads/attachment_data/file/926563/Framework_of_actions_to_contain_CPEdraft.pdf

7. Sypsa V, Psichogiou M, Bouzala G-A, Hadjihannas L, Hatzakis A, Daikos GL. Transmission dynamics of carbapenemase-producing Klebsiella pneumoniae and anticipated impact of infection control strategies in a surgical unit. PLoS ONE. 2012;7:e41068.

8. Knight GM, Dyakova E, Mookerjee S, Davies F, Brannigan ET, Otter JA, et al. Fast and expensive (PCR) or cheap and slow (culture)? A mathematical modelling study to explore screening for carbapenem resistance in UK hospitals. BMC Med. 2018;16:141.

9. Lapointe-Shaw L, Voruganti T, Kohler P, Thein H-H, Sander B, McGeer A. Cost-effectiveness analysis of universal screening for carbapenemaseproducing Enterobacteriaceae in hospital inpatients. Eur J Clin Microbiol Infect Dis. 2017;36:1047-55.

10. Bartsch SM, Wong KF, Mueller LE, Gussin GM, McKinnell JA, Tjoa T, et al. Modeling Interventions to Reduce the Spread of Multidrug-Resistant Organisms Between Health Care Facilities in a Region. JAMA Netw Open. 2021;4:e2119212.

11. Kardaś-Słoma L, Lucet J-C, Perozziello A, Pelat C, Birgand G, Ruppé E, et al. Universal or targeted approach to prevent the transmission of extendedspectrum beta-lactamase-producing Enterobacteriaceae in intensive care units: a cost-effectiveness analysis. BMJ Open. $2017 ; 7: \mathrm{e} 017402$. 
12. Pelat C, Kardaś-Słoma L, Birgand G, Ruppé E, Schwarzinger M, Andremont A, et al. Hand Hygiene, Cohorting, or Antibiotic Restriction to Control Outbreaks of Multidrug-Resistant Enterobacteriaceae. Infect Control Hosp Epidemiol. 2016;37:272-80.

13. Guyot, J.M. Etude sur l'évaluation des pratiques dans le cadre de la lutte contre les infections nosocomiales sur les «frictions hydro-alcooliques par spécialités médico-chirurgicales». Lot $\mathrm{n}^{\circ} 2$ : Enquête sur le nombre d’opportunités d’hygiène des mains par spécialité médico-chirugicale: Ministère de la Santé, de la Jeunesse et des Sports et de la vie associative, Direction Générale de la Santé, Sous-direction Prévention des risques infectieux; 2008.

14. Vanhems P, Barrat A, Cattuto C, Pinton J-F, Khanafer N, Régis C, et al. Estimating Potential Infection Transmission Routes in Hospital Wards Using Wearable Proximity Sensors. Viboud C, editor. PLoS ONE. 2013;8:e73970.

15. Direction de l'hospitalisation et de l'organisation des soins. Circulaire DHOS/02 no 2007-117 du 28 mars 2007 relative à la filière de soins gériatriques [Internet]. 2020 [cited 2020 Nov 13]. Available from: https://solidarites-sante.gouv.fr/fichiers/bo/2007/07-04/a0040058.htm

16. Jolivet S, Vaillant L, Poncin T, Lolom I, Gaudonnet Y, Rondinaud E, et al. Prevalence of carriage of extended-spectrum $\beta$-lactamase-producing enterobacteria and associated factors in a French hospital. Clin Microbiol Infect. 2018;24:1311-4.

17. Otter JA, Dyakova E, Bisnauthsing KN, Querol-Rubiera A, Patel A, Ahanonu C, et al. Universal hospital admission screening for carbapenemaseproducing organisms in a low-prevalence setting. J Antimicrob Chemother. 2016;71:3556-61.

18. Birgand G, Leroy C, Nerome S, Luong Nguyen LB, Lolom I, Armand-Lefevre L, et al. Costs associated with implementation of a strict policy for controlling spread of highly resistant microorganisms in France. BMJ Open. 2016;6:e009029.

19. European Centre for Disease Prevention and Control. Antimicrobial resistance interactive database (EARS-Net) [Internet]. 2016 [cited 2016 Sep 6]. Available from: http://ecdc.europa.eu/en/healthtopics/antimicrobial-resistance-and-

consumption/antimicrobial_resistance/database/Pages/database.aspx

20. Otter JA, Mookerjee S, Davies F, Bolt F, Dyakova E, Shersing Y, et al. Detecting carbapenemase-producing Enterobacterales (CPE): an evaluation of an enhanced CPE infection control and screening programme in acute care. Journal of Antimicrobial Chemotherapy. 2020;75:2670-6.

21. Saliba R, Neulier C, Seytre D, Fiacre A, Faibis F, Leduc P, et al. Can real-time polymerase chain reaction allow a faster recovery of hospital activity in cases of an incidental discovery of carbapenemase-producing Enterobacteriaceae and vancomycin-resistant Enterococci carriers? Journal of Hospital Infection. 2019;103:115-20.

22. Maechler F, Schwab F, Hansen S, Fankhauser C, Harbarth S, Huttner BD, et al. Contact isolation versus standard precautions to decrease acquisition of extended-spectrum $\beta$-lactamase-producing Enterobacterales in non-critical care wards: a cluster-randomised crossover trial. The Lancet Infectious Diseases. 2020;20:575-84.

23. Duval A, Obadia T, Boëlle P-Y, Fleury E, Herrmann J-L, Guillemot D, et al. Close proximity interactions support transmission of ESBL-K. pneumoniae but not ESBL-E. coli in healthcare settings. Lloyd-Smith J, editor. PLOS Computational Biology. 2019;15:e1006496.

24. Haverkate MR, Bootsma MCJ, Weiner S, Blom D, Lin MY, Lolans K, et al. Modeling Spread of KPC-Producing Bacteria in Long-Term Acute Care Hospitals in the Chicago Region, USA. Infection Control \& Hospital Epidemiology. 2015;36:1148-54.

25. Chia PY, Sengupta S, Kukreja A, S.L. Ponnampalavanar S, Ng OT, Marimuthu K. The role of hospital environment in transmissions of multidrugresistant gram-negative organisms. Antimicrob Resist Infect Control. 2020;9:29.

26. Jimenez A, Fennie K, Munoz-Price LS, Ibrahimou B, Pekovic V, Abbo LM, et al. Duration of carbapenemase-producing Enterobacteriales carriage among ICU patients in Miami, FL: A retrospective cohort study. Am J Infect Control. 2021;49:1281-6.

27. Otter JA, Burgess P, Davies F, Mookerjee S, Singleton J, Gilchrist M, et al. Counting the cost of an outbreak of carbapenemase-producing Enterobacteriaceae: an economic evaluation from a hospital perspective. Clin Microbiol Infect. 2017;23:188-96.

28. De Angelis G, Murthy A, Beyersmann J, Harbarth S. Estimating the impact of healthcare-associated infections on length of stay and costs. Clin Microbiol Infect. 2010;16:1729-35.

29. Birgand G, Schwarzinger M, Perozziello A, Lolom I, Pelat C, Armand-Lefevre L, et al. Prolonged hospital stay, an adverse effect of strict national policy for controlling the spread of highly resistant microorganisms. Infect Control Hosp Epidemiol. 2014;35:1427-9.

30. Schechner V, Kotlovsky T, Kazma M, Mishali H, Schwartz D, Navon-Venezia S, et al. Asymptomatic rectal carriage of blaKPC producing carbapenem-resistant Enterobacteriaceae: who is prone to become clinically infected? Clinical Microbiology and Infection. 2013;19:451-6.

31. ATIH-Agence technique de l'information sur l'hospitalisation. Analyse de l'activité hospitalière [Internet]. 2017 [cited 2019 Jan 15]. Available from: https://www.atih.sante.fr/sites/default/files/public/content/3478/synthese_aah_2017.pdf

Page $11 / 15$ 
32. Barbier F, Pommier C, Essaied W, Garrouste-Orgeas M, Schwebel C, Ruckly S, et al. Colonization and infection with extended-spectrum $\beta$-lactamaseproducing Enterobacteriaceae in ICU patients: what impact on outcomes and carbapenem exposure? J Antimicrob Chemother. 2016;71:1088-97.

33. Qureshi ZA, Paterson DL, Potoski BA, Kilayko MC, Sandovsky G, Sordillo E, et al. Treatment Outcome of Bacteremia Due to KPC-Producing Klebsiella pneumoniae: Superiority of Combination Antimicrobial Regimens. Antimicrob Agents Chemother. 2012;56:2108-13.

34. Derde LPG, Cooper BS, Goossens H, Malhotra-Kumar S, Willems RJL, Gniadkowski M, et al. Interventions to reduce colonisation and transmission of antimicrobial-resistant bacteria in intensive care units: an interrupted time series study and cluster randomised trial. Lancet Infect Dis. 2014;14:319.

35. Segagni Lusignani L, Presterl E, Zatorska B, Van den Nest M, Diab-Elschahawi M. Infection control and risk factors for acquisition of carbapenemase-producing enterobacteriaceae. A 5 year (2011-2016) case-control study. Antimicrobial Resistance \& Infection Control [Internet]. 2020 [cited 2020 Nov 20];9. Available from: https://aricjournal.biomedcentral.com/articles/10.1186/s13756-019-0668-2

36. Venier AG, Zaro-Goni D, Pefau M, Hauray J, Nunes J, Cadot C, et al. Performance of hand hygiene in 214 healthcare facilities in South-Western France. Journal of Hospital Infection. 2009;71:280-2.

37. Chaix C, Durand-Zaleski I, Alberti C, Brun-Buisson C. Control of endemic methicillin-resistant Staphylococcus aureus: a cost-benefit analysis in an intensive care unit. JAMA. 1999;282:1745-51.

38. Mollers M, Lutgens SP, Schoffelen AF, Schneeberger PM, Suijkerbuijk AWM. Cost of Nosocomial Outbreak Caused by NDM-1-Containing Klebsiella pneumoniae in the Netherlands, October 2015-January 2016. Emerging Infect Dis. 2017;23:1574-6.

39. Schwaber MJ, Lev B, Israeli A, Solter E, Smollan G, Rubinovitch B, et al. Containment of a country-wide outbreak of carbapenem-resistant Klebsiella pneumoniae in Israeli hospitals via a nationally implemented intervention. Clin Infect Dis. 2011;52:848-55.

40. Fournier S, Monteil C, Lepainteur M, Richard C, Brun-Buisson C, Jarlier V, et al. Long-term control of carbapenemase-producing Enterobacteriaceae at the scale of a large French multihospital institution: a nine-year experience, France, 2004 to 2012. Eurosurveillance. 2014;19:20802.

41. Cohen MJ, Block C, Levin PD, Schwartz C, Gross I, Weiss Y, et al. Institutional Control Measures to Curtail the Epidemic Spread of CarbapenemResistant Klebsiella pneumoniae: A 4-Year Perspective. Infect Control Hosp Epidemiol. 2011;32:673-8.

42. Santé Publique France. Bilan des signalements BHRe 2019 [Internet]. 2020. Available from: https://www.santepubliquefrance.fr/maladies-ettraumatismes/infections-associees-aux-soins-et-resistance-aux-antibiotiques/resistance-aux-antibiotiques/documents/donnees/bilan-bhre-2019

43. Srigley JA, Furness CD, Baker GR, Gardam M. Quantification of the Hawthorne effect in hand hygiene compliance monitoring using an electronic monitoring system: a retrospective cohort study. BMJ Qual Saf. BMJ Publishing Group Ltd; 2014;23:974-80.

44. Hagel S, Reischke J, Kesselmeier M, Winning J, Gastmeier P, Brunkhorst FM, et al. Quantifying the Hawthorne Effect in Hand Hygiene Compliance Through Comparing Direct Observation With Automated Hand Hygiene Monitoring. Infect Control Hosp Epidemiol. 2015;36:957-62.

45. Assistance publique - Hôpitaux de Paris (AP-HP). Base de données PMSI. 2015.

46. Swaminathan M, Sharma S, Blash SP, Patel G, Banach DB, Phillips M, et al. Prevalence and Risk Factors for Acquisition of Carbapenem-Resistant Enterobacteriaceae in the Setting of Endemicity. Infection Control \& Hospital Epidemiology. 2013;34:809-17.

47. Bartsch SM, McKinnell JA, Mueller LE, Miller LG, Gohil SK, Huang SS, et al. Potential economic burden of carbapenem-resistant Enterobacteriaceae (CRE) in the United States. Clinical Microbiology and Infection. 2017;23:48.e9-48.e16.

48. Daroukh A, Delaunay C, Bigot S, Ceci JM, Siddhoun N, Bukreyeva I, et al. Characteristics and costs of carbapenemase-producing enterobacteria carriers (2012/2013). Médecine et Maladies Infectieuses. 2014;44:321-6.

49. D’Agata EMC, Webb G, Horn M. A mathematical model quantifying the impact of antibiotic exposure and other interventions on the endemic prevalence of vancomycin-resistant enterococci. J Infect Dis. 2005;192:2004-11.

50. Almagor J, Temkin E, Benenson I, Fallach N, Carmeli Y, on behalf of the DRIVE-AB consortium. The impact of antibiotic use on transmission of resistant bacteria in hospitals: Insights from an agent-based model. Zhou Z, editor. PLOS ONE. 2018;13:e0197111.

51. Falagas ME, Tansarli GS, Karageorgopoulos DE, Vardakas KZ. Deaths attributable to carbapenem-resistant Enterobacteriaceae infections. Emerging Infect Dis. 2014;20:1170-5.

52. Austin DJ, Bonten MJ, Weinstein RA, Slaughter S, Anderson RM. Vancomycin-resistant enterococci in intensive-care hospital settings: transmission dynamics, persistence, and the impact of infection control programs. Proc Natl Acad Sci USA. 1999;96:6908-13.

Page 12/15 
53. Borer A, Saidel-Odes L, Eskira S, Nativ R, Riesenberg K, Livshiz-Riven I, et al. Risk factors for developing clinical infection with carbapenem-resistant Klebsiella pneumoniae in hospital patients initially only colonized with carbapenem-resistant K pneumoniae. American Journal of Infection Control. $2012 ; 40: 421-5$.

54. Tzouvelekis LS, Markogiannakis A, Piperaki E, Souli M, Daikos GL. Treating infections caused by carbapenemase-producing Enterobacteriaceae. Clinical Microbiology and Infection. 2014;20:862-72.

55. Adams DJ, Susi A, Nylund CM. Clinical characteristics, risk factors, and outcomes of patients hospitalized in the US military health system with carbapenem-resistant Enterobacteriaceae infection. American Journal of Infection Control. 2020;48:644-9.

56. Tumbarello M, Trecarichi EM, De Rosa FG, Giannella M, Giacobbe DR, Bassetti M, et al. Infections caused by KPC-producing Klebsiella pneumoniae: differences in therapy and mortality in a multicentre study. Journal of Antimicrobial Chemotherapy. 2015;70:2133-43.

57. Tato M, Ruiz-Garbajosa P, Traczewski M, Dodgson A, McEwan A, Humphries R, et al. Multisite Evaluation of Cepheid Xpert Carba-R Assay for Detection of Carbapenemase-Producing Organisms in Rectal Swabs. Carroll KC, editor. Journal of Clinical Microbiology. 2016;54:1814-9.

58. Dortet L, Fusaro M, Naas T. Improvement of the Xpert Carba-R Kit for the Detection of Carbapenemase-Producing Enterobacteriaceae. Antimicrobial Agents and Chemotherapy. 2016;60:3832-7.

59. Hoyos-Mallecot Y, Ouzani S, Dortet L, Fortineau N, Naas T. Performance of the Xpert ${ }^{\circledR}$ Carba-R v2 in the daily workflow of a hygiene unit in a country with a low prevalence of carbapenemase-producing Enterobacteriaceae. International Journal of Antimicrobial Agents. 2017;49:774-7.

60. Harris AD. Universal Glove and Gown Use and Acquisition of Antibiotic-Resistant Bacteria in the ICU: A Randomized Trial. JAMA [Internet]. 2013 [cited 2021 Jan 15]; Available from: http://jama.jamanetwork.com/article.aspx?doi=10.1001/jama.2013.277815

\section{Figures}

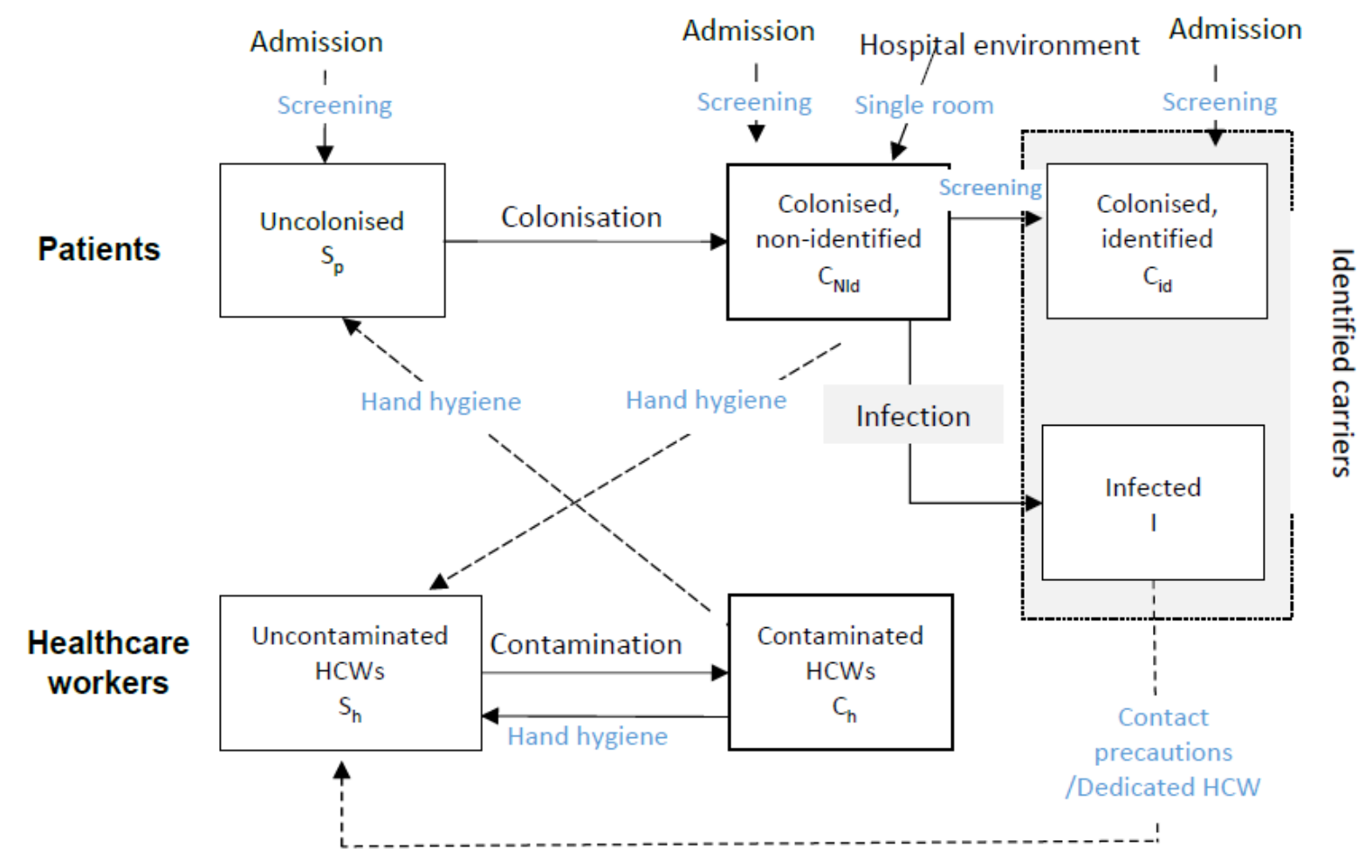

Figure 1

Flow diagram describing the CPE spread between patients and HCWs and the implementation of interventions. Possible interventions are indicated in blue: 1) hand hygiene, 2) contact precautions (better hand hygiene, gown and gloves), 3) dedicated staff (or cohorting of identified patients), 4) single room (limit the transmission by the environment), 5) screening on admission or during hospital stay. 


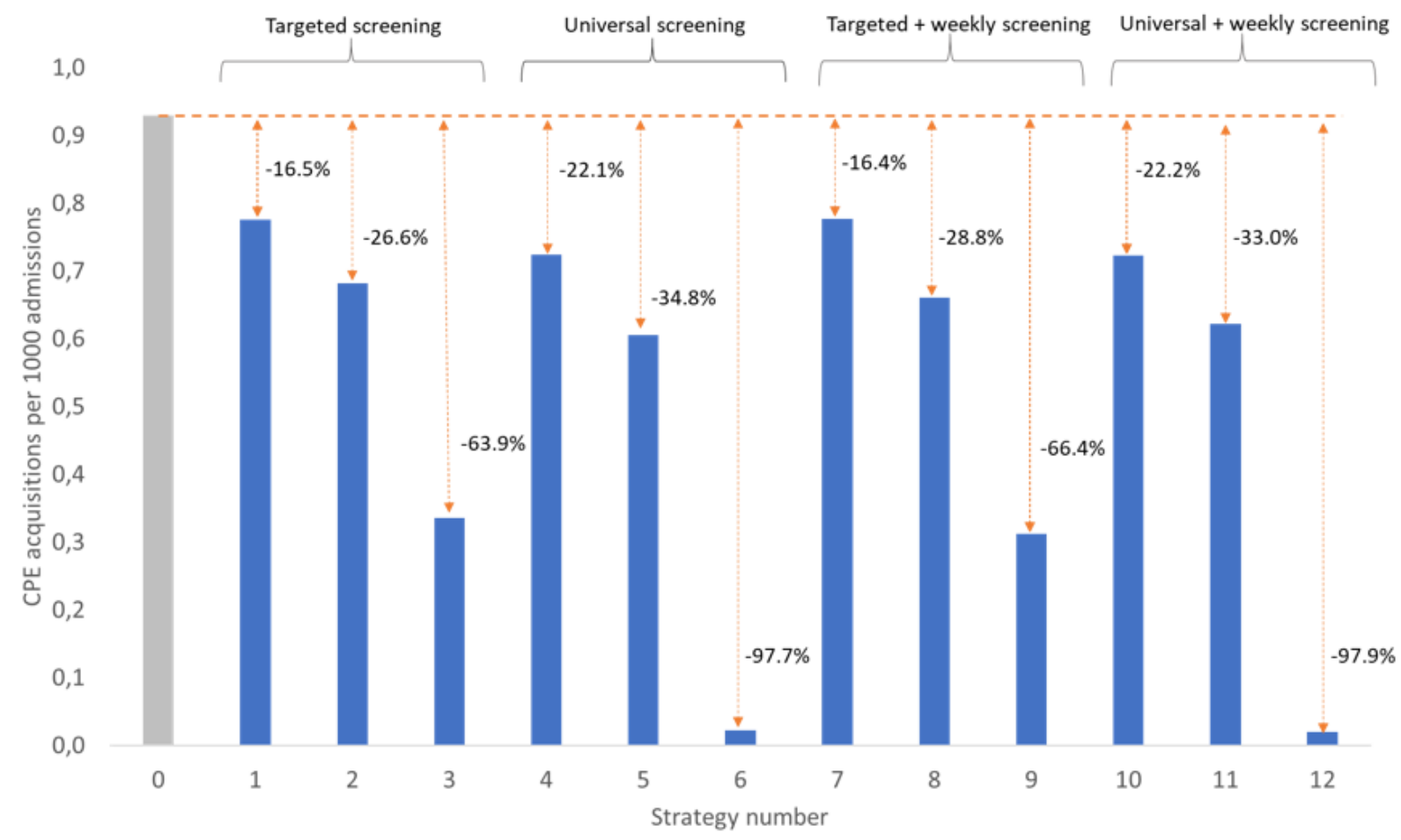

Figure 2

CPE acquisitions per 1000 admissions under different control strategies tested. Strategies: 0) standard contact precautions (baseline), 1) Targeted screening (TS) + contact precautions (CP) without isolation of carriers in single room, 2) TS + CP + single room, 3) TS + dedicated nursing staff (DNS) + single room, 4) Universal screening (US) + CP without isolation in single room, 5) US + CP + single room, 6) US + DNS + single room, 7) TS+ CP + without isolation of carriers in single room + weekly screening of contact patients (WSC), 8) TS + CP + single room + WSC, 9) TS + DNS + single room + WSC, 10) US+ CP without isolation in single room + WSC, 11) US + CP+ single room + WSC, 12) US+ DNS + single room + WSC.

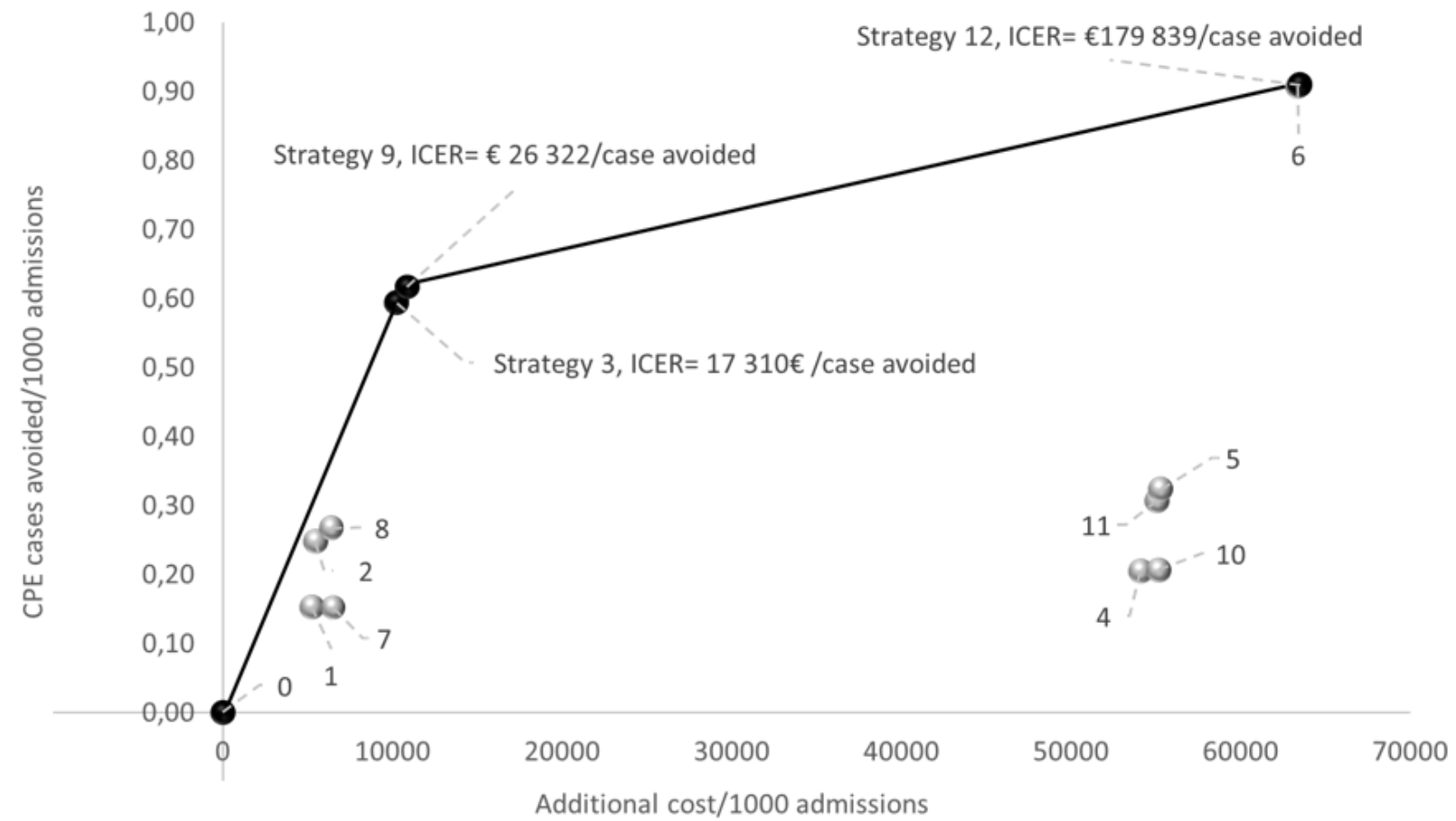

Figure 3 
Graphical representation of the efficiency frontier. The broken line visualizes the efficiency frontier: all interventions below the efficiency frontier are dominated.

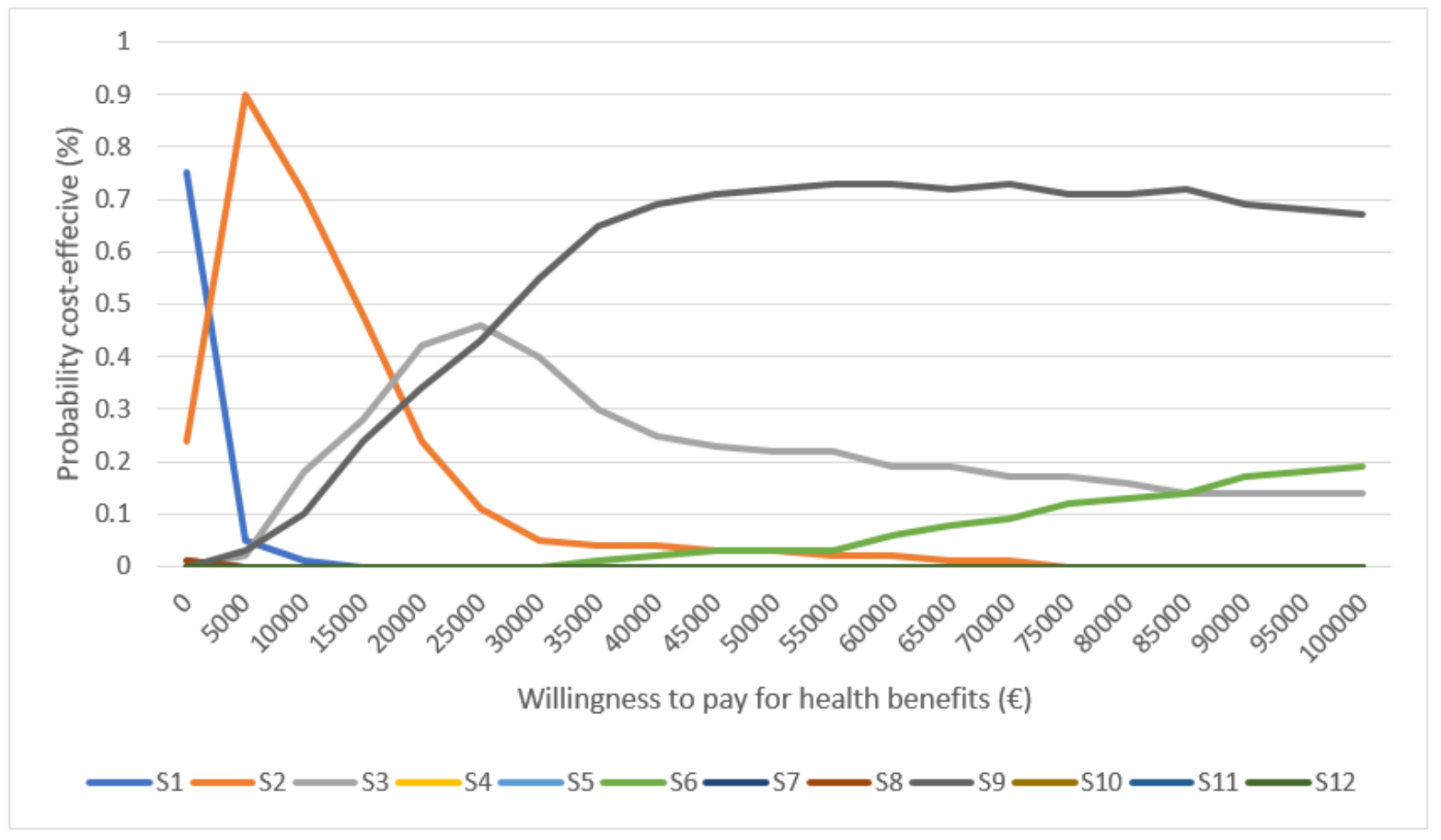

\section{Figure 4}

Cost-effectiveness acceptability curves for strategies. They represent the probability of each strategy to be cost-effective at different values of willingness to pay for a CPE case avoided. Strategies: 1) Targeted screening (TS) + contact precautions (CP) without isolation of carriers in single room, 2) TS + CP + single room, 3) TS + dedicated nursing staff (DNS) + single room, 4) Universal screening (US) + CP without isolation in single room, 5) $\mathrm{US}+\mathrm{CP}+$ single room , 6) US + DNS + single room, 7) TS + CP + without isolation of carriers in single room + weekly screening of contact patients (WSC), 8) TS + CP + single room + WSC, 9) TS + DNS + single room + WSC, 10) US + CP without isolation in single room + WSC, 11) US + CP+ single room + WSC, 12) US+ DNS + single room + WSC.

\section{Supplementary Files}

This is a list of supplementary files associated with this preprint. Click to download.
- AppendixA118.01.docx
- AppendixA218.01.docx 